\title{
Combined ARPES and STM study of Pb/Au(111) Moiré structure: One overlayer, two symmetries
}

\author{
A. Crepaldi, ${ }^{1, *}$ S. Pons, ${ }^{1,2}$ E. Frantzeskakis, ${ }^{3}$ F. Calleja, ${ }^{1}$ M. Etzkorn, ${ }^{1}$ A. P. Seitsonen, ${ }^{4}$ K. Kern, ${ }^{1,5}$ H. Brune, ${ }^{1}$ and M. Grioni ${ }^{1}$ \\ ${ }^{1}$ Institut de Physique de la Matière Condensée (ICPM), Ecole Polytechnique Fédérale de Lausanne (EPFL), \\ Station 3, CH-1005 Lausanne, Switzerland \\ ${ }^{2}$ Institut des NanoSciences de Paris (INSP), Université Pierre et Marie Curie (UPMC) - Paris 6, CNRS UMR 7588, France \\ ${ }^{3}$ Synchrotron SOLEIL, L'Orme des Merisiers, Saint Aubin-BP 48, 91192 Gif sur Yvette Cedex, France \\ ${ }^{4}$ Physikalisch-Chemisches Institut, University of Zürich, CH-8057 Zürich, Switzerland \\ ${ }^{5}$ Max-Plank-Institut für Festkörperforschung, D-70569, Stuttgart, Germany
}

(Received 15 February 2013; revised manuscript received 11 March 2013; published 27 March 2013)

\begin{abstract}
The structural and electronic properties of a $\mathrm{Pb}$ monolayer (ML) grown on $\mathrm{Au}(111)$ are investigated by scanning tunneling microscopy (STM) and angle resolved photoelectron spectroscopy (ARPES). We find an incommensurate Moiré structure with an approximate $(5.77 \times 5.77) \mathrm{R} 21.5^{\circ}$ unit cell, and two rotational domains. We observe three $\mathrm{Pb}$-derived bands of $p$ orbital character. The symmetry properties and sharpness of their Fermi surfaces are remarkably different. They reflect the different degrees of hybridization of these bands with the $\mathrm{Au}(111)$ bulk continuum.
\end{abstract}

DOI: 10.1103/PhysRevB.87.115138

PACS number(s): 73.20.At, 79.60.-i

\section{INTRODUCTION}

Angle resolved photoelectron spectroscopy (ARPES) is ideally suited to probe the influence of commensurate and incommensurate charge (spin) modulations on the electronic structure of solids. ${ }^{1-3}$ Characteristic signatures of superperiodicities have been observed by ARPES in bulk materials ${ }^{4-6}$ and at ordered interfaces. ${ }^{7-16}$ Lead overlayers on both metallic and semiconducting substrates are especially interesting in this respect. It is found that, due to their high lateral stiffness, monolayers (ML) of $\mathrm{Pb}$ form dense Moiré superstructures. The corrugation of the overlayer reduces the bonding strength to the substrate $^{17}$ and contributes to the remakable surfactant effect of $\mathrm{Pb}$ observed, e.g., in the homoepitaxial growth of $\mathrm{Cu}(111) .{ }^{18,19}$ ARPES was exploited to determine the band structure and the electron coherence length of a melting $\mathrm{Pb}$ layer on $\mathrm{Cu}(111){ }^{20}$ The unexpected observation of superconductivity in the 4/3 ML dense phase of $\mathrm{Pb}$ on $\mathrm{Si}(111)^{21,22}$ has further stimulated the interest for Pb-based interfaces.

Here we report scanning tunneling microscopy (STM) and low-energy electron diffraction (LEED) data revealing a two-domain incommensurate Moiré structure for a $\mathrm{Pb} \mathrm{ML}$ on $\mathrm{Au}(111)$. ARPES identifies three interface bands: two of them have (mainly) $\mathrm{Pb} p_{x y}$ character while the third one has $\mathrm{Pb} p_{z}$ character. The behavior of the two $\mathrm{Pb} p_{x y}$ states is remarkably different: one adjusts to the symmetry of the $\mathrm{Au}(111)$ substrate, whereas the other displays the symmetry of the two-domain modulated overlayer. The $p_{z}$ state is strongly broadened in energy and momentum, similar to previous observations for $\mathrm{Pb}$ MLs on $\mathrm{Ag}(111)^{7}$ and on $\mathrm{Cu}(111) .{ }^{18}$ These differences reflect the orbital-dependent hybridization of the three interface states with the continuum of substrate bulk states.

\section{EXPERIMENT}

The $\mathrm{Au}(111)$ substrate was cleaned by repeated sputtering (with $\mathrm{Ar}^{+}$at $300 \mathrm{~K}, 1 \mathrm{keV}$ for 30 minutes) and annealing (800 $\mathrm{K}$ for 25 minutes) cycles. In the ARPES experiments, the sharpness of the LEED pattern and of the spin-split
Shockley surface state were used as indicators of the order and cleanliness of the surface. $\mathrm{Pb}$ was evaporated on the substrate at $300 \mathrm{~K}$ (room temperature or RT) from a calibrated EFM3 Omicron evaporator, at a rate of $0.3 \mathrm{ML} / \mathrm{min}$. The structural quality of the interface, monitored by LEED, improved after a short post-annealing at $400 \mathrm{~K}$. ARPES mesurements were performed at RT with a Specs Phoibos 150 hemispherical analyzer. The experimental energy resolution was set to $10 \mathrm{meV}$. The UV light source was a monochromatized high-brightness Gammadata VUV 5000 lamp operating at the $\mathrm{He} \mathrm{I} \alpha(21.22 \mathrm{eV})$ line. Further ARPES measurements at liquid nitrogen temperature (not shown) indicate that no modification of the electronic properties of the interface occurs when varying the temperature in this range. STM experiments were performed in a separated UHV system equipped with a home-built $0.4 \mathrm{~K} \mathrm{STM.} \mathrm{In} \mathrm{the}$ measurements presented here the sample temperature was $4 \mathrm{~K}$, and the indicated bias voltage refers to the sample potential.

\section{RESULTS}

Large-scale STM images reveal that the $\mathrm{Pb}$ monolayer is ordered over large terraces. The constant-current STM image of Fig. 1(a) displays atomic resolution together with a long-range modulation, indicative of a Moiré structure. The periodicity of the overlayer is best determined from the Fourier transform map of Fig. 1(b), obtained from a larger $\left(20 \times 20 \mathrm{~nm}^{2}\right)$ STM image. It exhibits the hexagonal pattern of the $\mathrm{Pb} \mathrm{ML}$ (red circles), and the corresponding in-plane $\mathrm{Pb}-\mathrm{Pb}$ distance is essentially identical to the $\mathrm{Pb}$ bulk value of $3.50 \AA$. Additional spots associated with the Moiré structure define a hexagonal pattern (black circles) which is 4.74 times smaller than the $\mathrm{Pb}$ pattern, and rotated by $\beta=26.5^{\circ} \pm 1^{\circ}$. Complementary information is obtained from LEED which, unlike STM, also probes the underlying Au(111) substrate. Indeed, the most intense spots in the LEED pattern of Fig. 1(c) are the first-order diffraction spots of the Au substrate (green hexagon). Two smaller, identical patterns (blue and red hexagons) rotated by $\pm 5^{\circ}$, whose size corresponds to 

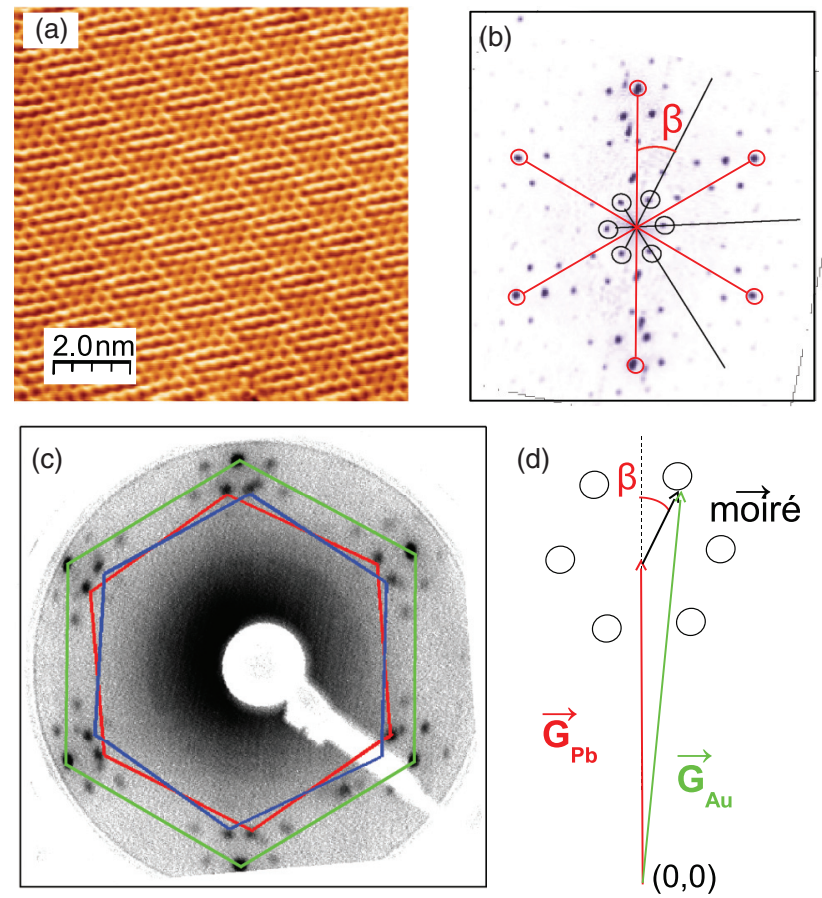

(e)

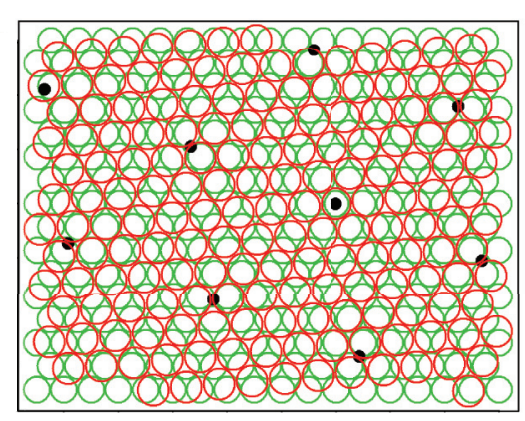

FIG. 1. (Color online) (a) Constant-current STM image of $1 \mathrm{ML}$ $\mathrm{Pb}$ on $\mathrm{Au}(111)$, showing one rotational domain of the Moiré structure $\left(V_{t}=2.0 \mathrm{~V}\right.$ and $I_{\mathrm{t}}=0.14 \mathrm{nA}, T=4.2 \mathrm{~K}$ ). (b) The Fourier transform of a larger $\left(20 \times 20 \mathrm{~nm}^{2}\right)$ STM image exhibits the hexagonal pattern (red circles) of a $\mathrm{Pb}$ ML and satellites corresponding to the Moiré superstructure spots (black circles). (c) LEED image showing the $\mathrm{Au}(111)$ (green) and two equivalent $\mathrm{Pb}$ (blue and red) hexagonal patterns, and Moiré satellites. (d) Reciprocal lattice vectors of the $\mathrm{Au}, \mathrm{Pb}$ (one domain), and Moiré patterns. (e) In-plane structural model of the interface (one domain). $\mathrm{Au}(\mathrm{Pb})$ atoms are represented by green (red) circles.

the periodicity of the $\mathrm{Pb} \mathrm{ML}$, can be identified inside the $\mathrm{Au}$ hexagon. Clearly, two equivalent rotational domains are simultaneously present in the $\mathrm{Pb}$ overlayer. Higher order diffraction spots show the good crystalline quality of the samples studied by ARPES. The relation between the substrate and overlayer periodicities, as determined from $\mathrm{STM}^{23}$ and LEED, is best seen by the reciprocal lattice vectors shown in Fig. 1(d). The Moiré unit cell is approximately given by $(5.77 \times 5.77) \mathrm{R} 21.5^{\circ}$ with respect to the $\mathrm{Au}(111)$ substrate. It is compatible with a rotation of $\pm 5^{\circ}$ between the $\mathrm{Pb}$ and the $\mathrm{Au}(111)$ lattices; ${ }^{24}$ see the ball model in Fig. 1(e). The good agreement between the low-temperature (4 K) STM data and the room-temperature LEED data indicates
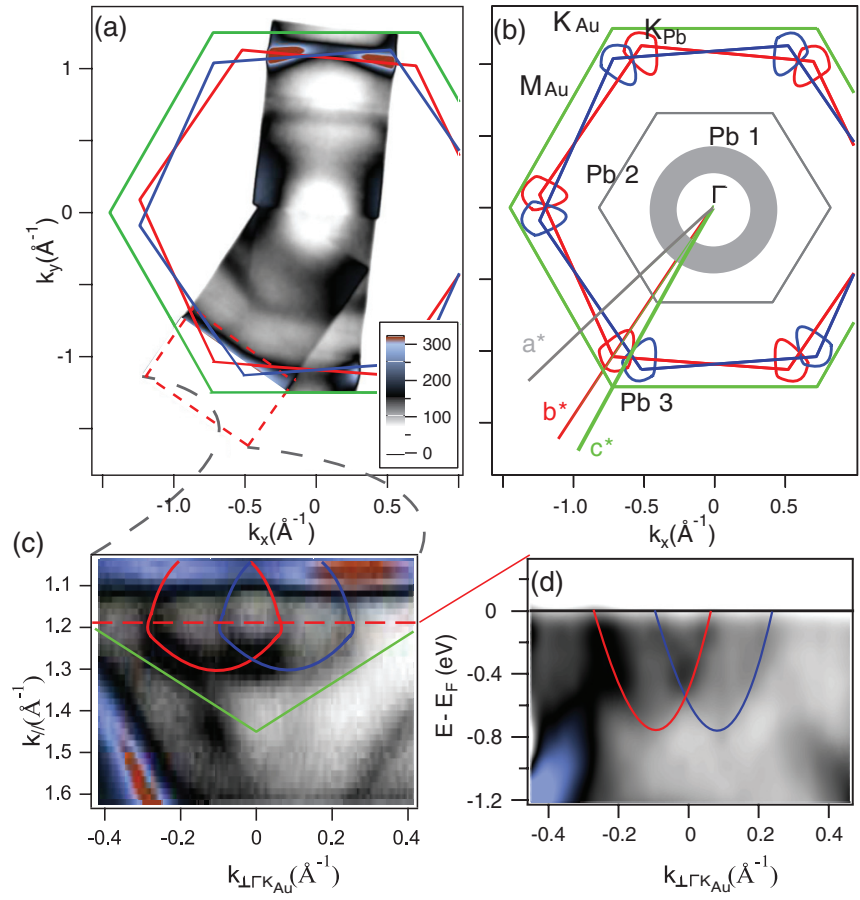

FIG. 2. (Color online) (a) ARPES Fermi surface (FS) map. Hexagons outline the $\mathrm{Au}(111)$ surface BZ (green) and the BZs of the two $\mathrm{Pb}$ domains (red and blue). The FS sheets of the three $\mathrm{Pb}$-derived bands are schematically illustrated in (b). (c) Closeup of the area within the dashed rectangle in (b); red and blue lines outline the $\mathrm{Pb} 3$-derived pockets for the two $\mathrm{Pb}$ domains. (d) $E$ vs $k$ dispersion of $\mathrm{Pb} 3$ (two domains) along the dashed line in (c), perpendicular to $\overline{\Gamma K}_{\mathrm{Au}}$.

that, despite the strong electron-phonon coupling of $\mathrm{Pb}$, the structure of this interface does not vary in this temperature range.

We now turn to the ARPES data, and to the experimental band structure arising from the interaction of the $\mathrm{Pb} \mathrm{ML}$ with the substrate. Figure 2(a) shows a portion of the Fermi surface (FS), extracted from ARPES measurements along the $\overline{\Gamma K}$ and two inequivalent $\overline{\Gamma M}$ high symmetry directions. The green hexagon is the $\mathrm{Au}(111)$ surface Brillouin zone (BZ); the smaller red and blue hexagons define the Brillouin zones of a $\mathrm{Pb}$ monolayer rotated by $\pm 5^{\circ}$. The most intense feature, near the Au BZ boundary, originates from the bulk Au $s p$ valence band. All the remaining features are $\mathrm{Pb}$ derived, and are schematically represented in Fig. 2(b). Previous tight-binding calculations performed for dense $\mathrm{Pb}$ monolayers on $\mathrm{Cu}(111)$ and $\operatorname{Ag}(111),{ }^{7,18,20}$ found three $\mathrm{Pb}$-derived bands: a band with $p_{z}$ orbital character with the smallest Fermi wave vector, hereafter labeled $\mathrm{Pb} 1$, and two bands with $p_{x y}$ character, labeled $\mathrm{Pb} 2$ and $\mathrm{Pb} 3$. The same picture is also consistent with our data, namely with the multisheet Fermi surface of Fig. 2(a). The diffuse, almost circular contour around the $\bar{\Gamma}$ point is attributed to $\mathrm{Pb} 1$, by analogy with the $\mathrm{Cu}(111)$ and $\mathrm{Ag}(111)$ cases. $\mathrm{Pb} 2$ forms a larger hexagonal Fermi surface. This single hexagonal contour is aligned with the BZ of the substrate. The symmetry of the FS associated with the third band is remarkably different. Pb3 crosses the Fermi level $\left(E_{\mathrm{F}}\right)$ near the corners of the $\mathrm{Pb} \mathrm{BZ}$, as illustrated in panel (c), 

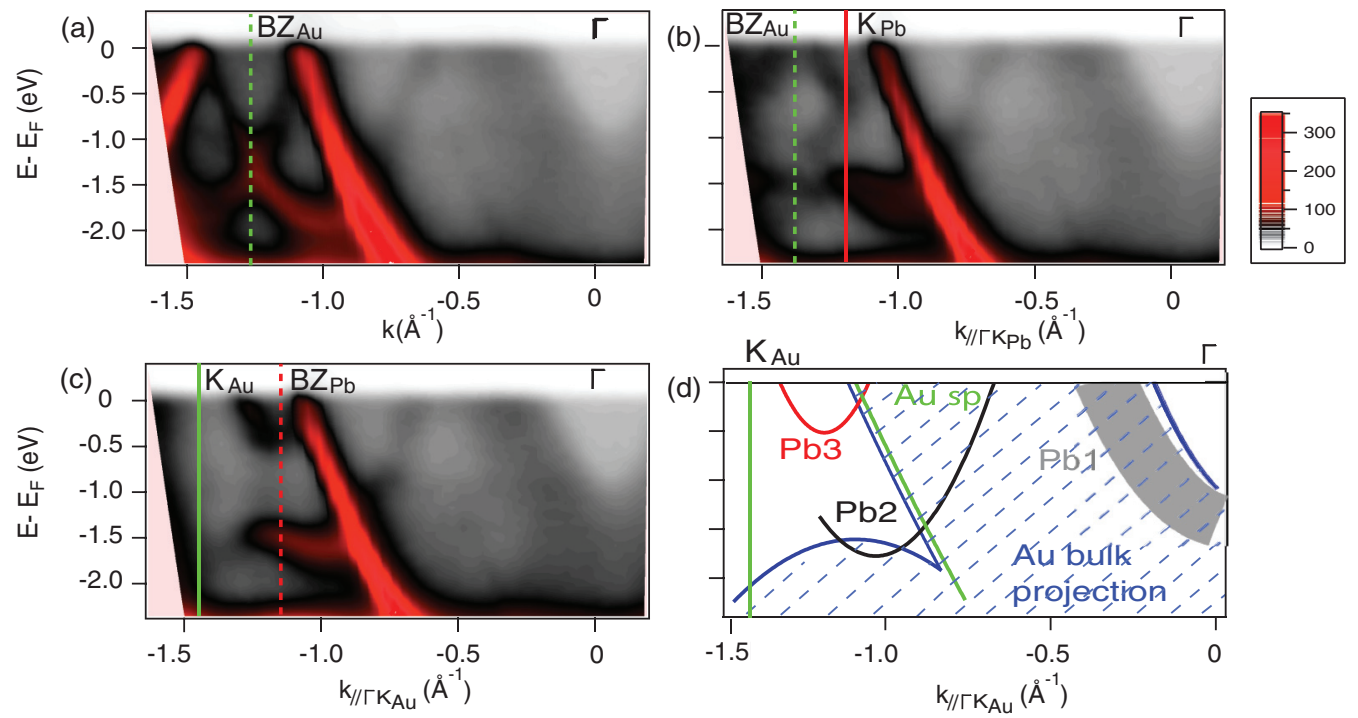

FIG. 3. (Color online) ARPES band dispersion along (a) a line $15^{\circ}$ from $\overline{\Gamma K}_{\mathrm{Au}}$ [line $\mathrm{a}^{*}$ in Fig. 2(b)]; (b) $\overline{\Gamma K}_{\mathrm{Pb}}$ [line b* in Fig. 2(b)]; and (c) $\overline{\Gamma K}_{\mathrm{Au}}$ [line $\mathrm{c}^{*}$ in Fig. 2(b)]. Vertical lines (solid lines for high-symmetry points) indicate the BZ boundaries. The experimental dispersion (c) is schematically illustrated in (d), where the calculated Au band structure projected on the (111) surface from Ref. 25 is also shown.

which shows a closeup of the area within the dashed rectangle in Fig. 2(a). It forms a trigonally distorted pocket around the corners $\left(\overline{\mathrm{K}}_{\mathrm{Pb}}\right)$ of the $\mathrm{BZ}$ of both $\mathrm{Pb}$ rotational domains. The adjacent pockets partially overlap with each other, and also with the substrate FS, the strongest feature in panel (c). Figure 2(d) illustrates the nearly parabolic $E$ vs $k$ dispersion of the overlapping $\mathrm{Pb} 3$ electron pockets along the dashed line in panel (c), perpendicular to the $\overline{\Gamma K}_{\mathrm{Au}}$ direction.

Figure 3 presents the experimental band structure over a broad momentum range, along the three lines marked $a^{*}, b^{*}$, c* in Fig. 2(b). They correspond to (a) a line $15^{\circ}$ from $\overline{\Gamma K}_{\mathrm{Au}}$, (b) the $\overline{\Gamma K}_{\mathrm{Pb}}$ direction for one domain, and (c) the $\overline{\Gamma K}_{\mathrm{Au}}$ direction $\left(\overline{\Gamma \mathrm{K}}_{\mathrm{Au}}=1.45 \AA^{-1} ; \overline{\Gamma \mathrm{K}}_{\mathrm{Pb}}=1.19 \AA^{-1}\right)$. A sketch of the experimental ARPES dispersion along $\overline{\Gamma K}_{\mathrm{Au}}$ is shown in panel (d), which also includes the projection of the calculated Au bulk band structure onto the (111) surface. ${ }^{25}$ Two of the $\mathrm{Pb}$-derived bands $(\mathrm{Pb} 1$ and $\mathrm{Pb} 2)$ and the dominant signal from the Au $s p$ band, are visible in panel (a). Pb1, which disperses upward from $\bar{\Gamma}$, lies entirely within the continuum of the $\mathrm{Au}$ bulk states. The bottom of this band around $-1.5 \mathrm{eV}$ and its Fermi level crossing near $k_{\mathrm{F} 1}=-0.35 \AA^{-1}$ are poorly defined, because its spectral weight is diffuse both in energy and momentum, similar to the case of $\mathrm{Pb} / \mathrm{Ag}(111),{ }^{7}$ and consistent with the blurred circular FS contour of Fig. 2. Pb2 exhibits a nearly parabolic upward dispersion from its minimum at $k_{\|}=1 \AA^{-1}$ and $-1.9 \mathrm{eV}$. Its intensity is strongly reduced after crossing the Au $s p$ band and entering the projected bulk continuum. The Fermi level crossing at $k_{\|} \sim-0.6 \AA^{-1}$ is part of the single hexagonal contour of Fig. 2(b). Folding of $\mathrm{Pb} 2$ and of the Au $s p$ band around the Au BZ boundary generates clear replicas of these bands. The $\mathrm{Pb} 3$ band is visible near $\mathrm{K} \mathrm{Pb}$ along lines $b^{*}$ [panel (b)] and c* [panel (c)] which intersect the electron pockets. The dispersion of $\mathrm{Pb} 2$ is strongly modified with respect to panel (a) beyond the Pb BZ boundary. A gap opens between $\mathrm{Pb} 2$ and $\mathrm{Pb} 3$, indicating an interaction between the two bands.
Neither the FS of Fig. 2 nor the bands of Fig. 3 exhibit replicas with the periodicity of the Moiré structure. This contrasts, e.g., with recent observations for graphene on a metallic substrate, ${ }^{15,16}$ where the Moiré structure gives rise to folded bands, with intensities proportional to the strength of the superlattice potential. ${ }^{7,18}$ Therefore, we conclude that the Moiré potential has a comparatively smaller amplitude in the present case.

\section{DISCUSSION}

The interaction between the $\mathrm{Pb}$ overlayer and the $\mathrm{Au}(111)$ substrate, and their different periodicities, are at the origin of a nontrivial band structure. We have performed a model tight-binding (TB) calculation as a guide to the interpretation of the ARPES results, namely to gain insight on the origin of the gap between the $\mathrm{Pb} 2$ and $\mathrm{Pb} 3$ bands observed in Fig. 3 . As a first step, we model a free-standing $\mathrm{Pb}$ overlayer by a two-dimensional hexagonal lattice, with three $p$ orbitals per site. The $p_{x}$ and $p_{y}$ orbitals couple in-plane to nearest neighbors via directional cosines, and give rise to two hybrid $p_{x y}$ bands, which are orthogonal to the $p_{z}$ band due to $+z /-z$ inversion symmetry. Model parameters quantify the Slater-Koster overlap integral ${ }^{26}$ (see Appendix). The only free parameter is $(p p \pi / p p \sigma)$. Similar oversimplified models have already been used to analyze the electronic structure of the $\mathrm{Pb} / \mathrm{Ag}(111)$ and $\mathrm{Pb} / \mathrm{Cu}(111)$ interfaces. ${ }^{7,18}$ The band structure shown in Fig. 4(a) reproduces the results of the previous studies. In particular, as expected, there is no interaction gap between the $p_{x y}$ and $p_{z}$ states and, at variance with experiment, between the two $p_{x y}$ states at the $\overline{\mathrm{K}}$ point.

A possible source of hybridization between the $\mathrm{Pb}$ bands is via the interaction with the substrate, which breaks the $+z /-z$ inversion symmetry. This interaction may be driven by the buckling of the $\mathrm{Pb}$ layer, as already proposed for the $\mathrm{Pb} / \mathrm{Cu}(111)$ interface. ${ }^{18}$ Following Ref. 27 we have modeled 

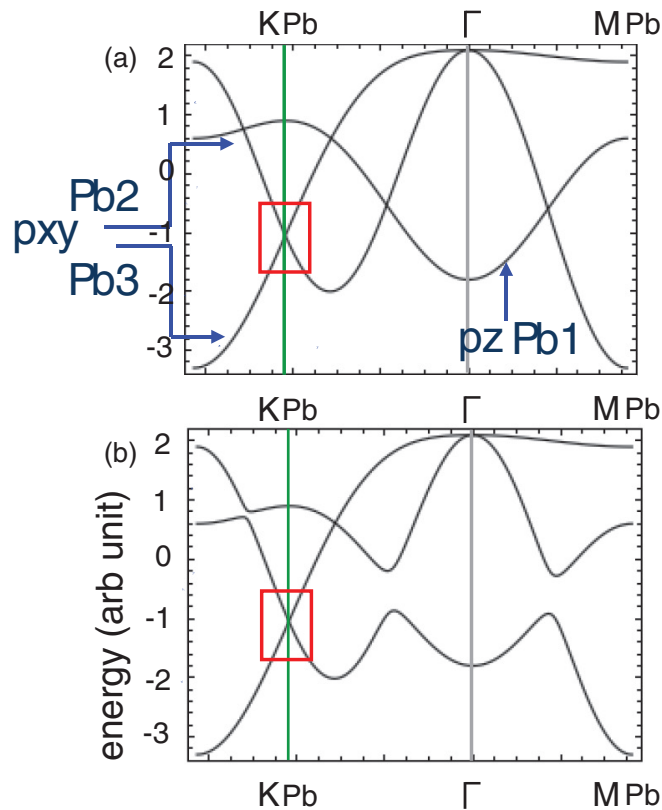

(c)

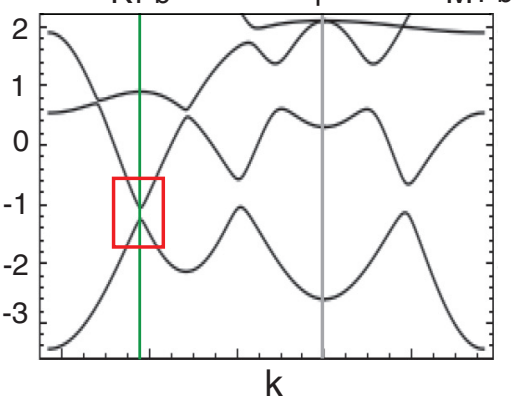

FIG. 4. (Color online) (a) Calculated TB bands for $p$ states on a free-standing hexagonal $\mathrm{Pb}$ monolayer. Two bands $\left(p_{x y}\right)$ have hybrid $p_{x}$ and $p_{y}$ orbital character; the third has $p_{z}$ character. (b) A gap opens between the $p_{x y}$ and $p_{z}$ states if the $+z /-z$ symmetry is broken. (c) The degeneracy of the two $p_{x y}$ states at $\overline{\mathrm{K}}$ is lifted when the sixfold in-plane symmetry is also broken by the interaction with the substrate. The parameters of the TB model are summarized in the Appendix.

in Fig. 4(b) the presence of the substrate by introducing an asymmetry in the $p_{z}$ state. The $p_{x y}$ and $p_{z}$ bands now hybridize, and gaps appear in correspondence of the avoided crossings. Nevertheless, the $p_{x y}$ states remain degenerate at $\overline{\mathrm{K}}$. The degeneracy is a necessary consequence of the artificial sixfold symmetry of the free-standing ML, which is broken by explicitly introducing an interaction with the substrate. We have extended the TB model to include an interaction between the $\mathrm{Pb} p$ orbitals and an $s$ orbital of the substrate, with three substrate atoms placed in hollow sites around each $\mathrm{Pb}$ atom. Figure 4(c) shows that in this case a gap, proportional to the $s p$ hybridization, opens between the $p_{x y}$ states at the $\overline{\mathrm{K}}$ point, as observed by ARPES. Moreover, the interaction with the substrate naturally breaks the $+z /-z$ inversion symmetry, and therefore opens a hybridization gap between the $p_{x y}$ and $p_{z}$ states, without any ad hoc asymmetry parameter. Such a gap was previously reported for $\mathrm{Pb} / \mathrm{Cu}(111) .{ }^{18}$ It cannot be experimentally observed here, because the (avoided) crossing of the two bands lies above the Fermi level.
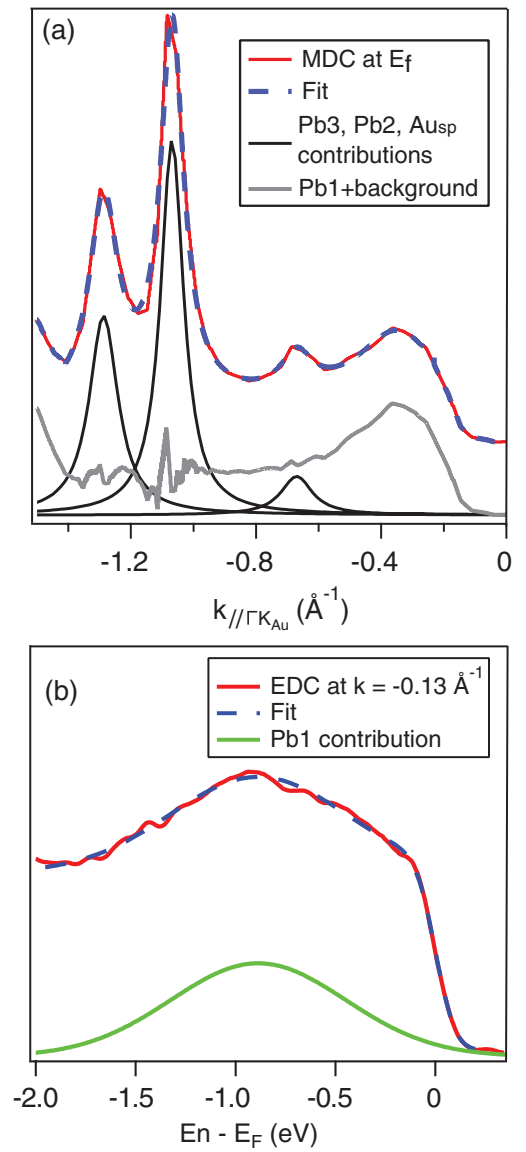

FIG. 5. (Color online) (a) Momentum distribution curve (MDC) at $E_{\mathrm{F}}$ along $\overline{\Gamma K}_{\mathrm{Au}}$. The widths (FWHM) of the Lorentzian peaks used in the fit are $0.11 \AA^{-1}(\mathrm{~Pb} 2), 0.11 \AA^{-1}(\mathrm{~Pb} 3)$, and $s p$ $0.08 \AA^{-1}$ (Au $s p$ ). The fit residual (grey) describes the remaining $\mathrm{Pb} 1$ peak and the background related to the $\mathrm{Au}$ bulk continuum. (b) The energy distribution curve (EDC) corresponding to $k=$ $-0.13 \AA^{-1}$. A Gaussian fit of the Pb1 state yields $\Delta E=1.1 \pm 0.1 \mathrm{eV}$ (FWHM).

The diffuse spectral weight distribution of the $\mathrm{Pb} 1$ band is a distinctive and unusual feature of the ARPES data of Fig. 3. This aspect is further illustrated in Fig. 5. Figure 5(a) shows a momentum distribution curve (MDC) extracted at $E_{\mathrm{F}}$, along the $\overline{\Gamma K}_{\mathrm{Au}}$ direction. The peaks corresponding to the $\mathrm{Pb} 2, \mathrm{~Pb} 3$, and the Au $s p$ bands were fitted by Lorentzians. The fit residual (grey) describes the remaining $\mathrm{Pb} 1$ band and the background related to the Au bulk continuum. This background reflects indirect transitions, and is proportional to the integrated density of states along $k_{z}{ }^{28}$ Remarkably, the momentum width of the $\mathrm{Pb} 1$ peak is 4-5 times broader than those of the other band features. Figure 5(b) gives a complementary view along the energy axis. It shows an energy distribution curve (EDC) corresponding to a wave vector $k=-0.13 \AA^{-1}$, where the $\mathrm{Pb} 1$ contribution is dominant. The spectrum is reproduced by a Gaussian line shape plus a constant to mimic the $\mathrm{Au}$ continuum, times a Fermi-Dirac cutoff; the additional $10 \mathrm{meV}$ experimental broadening is entirely negligible. The fit yields for $\mathrm{Pb} 1$ an energy width $\Delta E=1.1 \pm 0.1 \mathrm{eV}$ (full width at half maximum, FWHM), which is anomalously large for a quasiparticle peak, but comparable to the width 
of the corresponding $\mathrm{Pb} p_{z}$ band measured for a $\mathrm{Pb} \mathrm{ML}$ on $\operatorname{Ag}(111){ }^{7}$

What is the origin of the anomalous momentum and energy broadening? Due to the Moiré modulation, the $\mathrm{Pb}$ atoms sit in inequivalent sites, and the energies of the $\mathrm{Pb} p$ orbitals, especially the energies of the $p_{z}$ states which point towards the substrate, become site dependent. This effective disorder contributes to the spectral line widths, but quantitative simulations show that it cannot account for the large observed broadening. ${ }^{7}$ A different, more effective broadening mechanism is triggered by the interaction between the $\mathrm{Pb} p_{z}$ orbitals and the Au surface state. The Au surface state extends further into the substrate than the overlayer $p_{z}$ band. Through the hybridization with the surface state the $p_{z}$ state can then more effectively couple with the Au bulk continuum of states, over a large range of $k_{z}$ values that is inversely proportional to its (small) spatial extension in the $z$ direction. For each value of the $k_{x, y}$ surface wave vector, the $p_{z}$ state can essentially hybridize with the whole bulk continuum, thus acquiring an unusually large energy width. The large broadening is therefore the result of several factors: the nearly localized character of $p_{z}$ yielding a large uncertainty on $k_{z}$; the good spatial overlap with the Au surface state; and the fact that the $p_{z}$ band is entirely embedded in the bulk continuum.

Finally, we address the most striking aspect of the ARPES data, namely the different symmetries of the Fermi surface contours of the two $p_{x y}$ derived bands of Fig. 2. Pb3 forms two sets of FS pockets, one for each rotational domain, around the corners $\left(\overline{\mathrm{K}}_{\mathrm{Pb}}\right)$ of the $\mathrm{Pb} \mathrm{BZ}$, as expected for a band confined within the overlayer. By contrast, $\mathrm{Pb} 2$ forms a single hexagonal FS contour, aligned with the substrate BZ. How can two bands with the same origin exhibit such contrasting behavior? Fundamental principles require that all electronic states reflect the symmetry properties of the system, but such very general prescription cannot be strictly applied to our problem. The overall system has no clearly defined symmetry due to the coexisting incommensurate periodicities of overlayer and substrate. In similar circumstances, the simple notion of bands fades, and the relevant physical quantity is the spectral weight, which is distributed in energy and momentum space according to the strengths of the relevant interactions. ${ }^{3}$ In this perspective, the data of Fig. 2 demonstrate a different degree of hybridization of the two $p_{x y}$ bands with the substrate. The hybridization with $\mathrm{Pb} 2$ is stronger because this band is largely degenerate with the bulk continuum, namely near its Fermi surface crossing. Moreover, our TB results show that
$\mathrm{Pb} 2$ hybridizes with $\mathrm{Pb} 1$, thus acquiring some $p_{z}$ character, which also contributes to enhance the interaction of $\mathrm{Pb} 2$ with the substrate. The reduction of spectral intensity when $\mathrm{Pb} 2$ enters the continuum clearly indicates a sizable interaction. As a result, $\mathrm{Pb} 2$ strongly "feels" the potential of the substrate and conforms to its symmetry. $\mathrm{Pb} 3$, on the other hand, lies within the projected bulk band gap, and its interaction with the substrate is very weak. Therefore it remains confined within the overlayer and exhibits the symmetry of the two domains.

\section{CONCLUSIONS}

We have studied the structural and electronic properties of a Pb ML on $\mathrm{Au}(111)$ by STM and ARPES. We observed an incommensurate Moiré modulation, which is typical of $\mathrm{Pb}$ MLs on the (111) noble metal surfaces. We modeled the experimental band structure by a simple tight-binding model, which illustrates the importance of the overlayer-substrate interactions. The electronic structure of the interface is shaped by the different degrees of hybridization of the three $\mathrm{Pb}$ derived $p$ states. It varies from very strong $(\mathrm{Pb} 1)$ to very weak $(\mathrm{Pb} 3)$, and determines the energy-momentum spectral weight distribution, and the symmetry of the Fermi surface contours.

\section{ACKNOWLEDGMENT}

This work was supported by the Swiss NSF and by the MaNEP NCCR.

\section{APPENDIX}

The hybridization parameters used in the tight binding model presented in Fig. 4 are summarized in Table I.

TABLE I. Hybridization parameters following the notation of Ref. 26 in units of $(p p \sigma)$. Any proportional modification of all relevant parameters affects only the energy scaling.

\begin{tabular}{lccc}
\hline \hline Parameters & Fig. 4(a) & Fig. 4(b) & Fig. 4(c) \\
\hline$(p p \sigma)$ & 1 & 1 & 1 \\
$(p p \pi)$ & 0.3 & 0.3 & 0.3 \\
$(s s \sigma)$ & N/A & N/A & 0.5 \\
$(s p \sigma)$ & N/A & N/A & 0.5 \\
$z$ asymmetry & 0 & 0.1 & 0 \\
\hline \hline
\end{tabular}

*alberto.crepaldi@epfl.ch

${ }^{1}$ A. Damascelli, Z. Hussain, and Z.-H. Shen, Rev. Mod. Phys. 75, 473 (2003).

${ }^{2}$ M. Grioni, C. R. Ast, D. Pacilé, M. Papagno, H. Berger, and L. Perfetti, New J. Phys. 7, 106 (2005).

${ }^{3}$ J. Voit, L. Perfetti, F. Zwick, H. Berger, G. Margaritondo, G. Grüner, H. Hochst, and M. Grioni, Science 290, 501 (2000).

${ }^{4}$ J. Schäfer, E. Rotenberg, G. Meigs, S. D. Kevan, P. Blaha, and S. Hüfner, Phys. Rev. Lett. 83, 2069 (1999).
${ }^{5}$ T. Yokoya, T. Kiss, A. Chainani, S. Shin, and K. Yamaya, Phys. Rev. B 71, 140504 (2005).

${ }^{6}$ L. X. Yang, Y. Zhang, H. Ou, J. F. Zhao, D. Shen, B. Zhou, J. Wei, F. Chen, M. Xu, C. He et al., Phys. Rev. Lett. 102, 107002 (2009).

${ }^{7}$ C. R. Ast, D. Pacilé, M. Papagno, T. Gloor, F. Mila, S. Fedrigo, G. Wittich, K. Kern, H. Brune, and M. Grioni, Phys. Rev. B 73, 245428 (2006).

${ }^{8}$ P. Moras, W. Theis, L. Ferrari, S. Gardonio, J. Fujii, K. Horn, and C. Carbone, Phys. Rev. Lett. 96, 156401 (2006). 
${ }^{9}$ T. Brugger, S. Günther, B. Wang, H. Dil, M.-L. Bocquet, J. Osterwalder, J. Wintterlin, and T. Greber, Phys. Rev. B 79, 045407 (2009).

${ }^{10}$ M. Corso, W. Auwärter, M. Muntwiler, A. Tamai, T. Greber, and J. Osterwalder, Science 303, 217 (2004).

${ }^{11}$ R. Laskowski, P. Blaha, T. Gallauner, and K. Schwarz, Phys. Rev. Lett. 98, 106802 (2007).

${ }^{12}$ A. T. N'Diaye, S. Bleikamp, P. J. Feibelman, and T. Michely, Phys. Rev. Lett. 97, 215501 (2006).

${ }^{13}$ S. Marchini, S. Günther, and J. Wintterlin, Phys. Rev. B 76, 075429 (2007).

${ }^{14}$ T. Land, T. Michely, R. Behm, J. Hemminger, and G. Comsa, Surf. Sci. 264, 261 (1992).

${ }^{15}$ I. Pletikosic, M. Kralj, P. Pervan, R. Brako, J. Coraux, A. T. N'Diaye, C. Busse, and T. Michely, Phys. Rev. Lett. 102, 056808 (2009).

${ }^{16}$ S. Rusponi, M. Papagno, P. Moras, S. Vlaic, M. Etzkorn, P. M. Sheverdyaeva, D. Pacilé, H. Brune, and C. Carbone, Phys. Rev. Lett. 105, 246803 (2010).

${ }^{17}$ N. Tsud, S. Fabik, V. Dudr, M. Vondracek, V. Chab, V. Matolin, and K. Prince, Surf. Sci. 542, 112 (2003).
${ }^{18}$ F. Baumberger, A. Tamai, M. Muntwiler, T. Greber, and J. Osterwalder, Surf. Sci. 532-535, 82 (2003).

${ }^{19}$ J. Camarero, J. Ferrón, V. Cros, L. Gómez, A. L. Vazquez de Parga, J. M. Gallego, J. E. Prieto, J. J. de Miguel, and R. Miranda, Phys. Rev. Lett. 81, 850 (1998).

${ }^{20} \mathrm{~F}$. Baumberger, W. Auwärter, T. Greber, and J. Osterwalder, Science 306, 2221 (2004).

${ }^{21}$ S. Qin, J. Kim, Q. Niu, and C.-K. Shih, Science 324, 1314 (2009).

${ }^{22}$ T. Zhang, P. Cheng, W.-J. Li, Y.-J. Sun, G. Wang, X.-G. Zhu, K. He, L. Wang, X. Ma, X. Chen et al., Nat. Phys. 6, 104 (2010).

${ }^{23}$ I. Horcas, R. Fernández, J. M. Gmez-Rodríguez, J. Colchero, J. Gmez-Herrero, and A. M. Baro, Rev. Sci. Instrum. 78, 013705 (2007).

${ }^{24}$ T. Wiederholt, H. Brune, J. Wintterlin, R. Behm, and G. Ertl, Surf. Sci. 324, 91 (1995).

${ }^{25}$ R. Mazzarello, A. D. Corso, and E. Tosatti, Surf. Sci. 602, 893 (2008).

${ }^{26}$ J. C. Slater and G. F. Koster, Phys. Rev. 94, 1498 (1954).

${ }^{27}$ L. Petersen and P. Hedegard, Surf. Sci. 459, 49 (2000).

${ }^{28}$ M. Lindroos and A. Bansil, Phys. Rev. Lett. 77, 2985 (1996). 